\title{
Pacific
}

Journal of

Mathematics

\section{CONFORMAL INVARIANTS ASSOCIATED TO A MEASURE: CONFORMALLY COVARIANT OPERATORS}

Sun-Yung A. Chang, Matthew J. Gursky and Paul Yang 


\title{
CONFORMAL INVARIANTS ASSOCIATED TO A MEASURE: CONFORMALLY COVARIANT OPERATORS
}

\author{
Sun-Yung A. Chang, Matthew J. Gursky and Paul Yang
}

\begin{abstract}
We study Riemannian manifolds $\left(M^{n}, g\right)$ equipped with a smooth measure $m$. We show that the construction of conformally covariant operators of Graham-Jenne-Mason-Sparling can be adapted to this setting. As a byproduct, we define a family of scalar curvatures, one of which corresponds to Perelman's scalar-curvature function. We also study the variational problem naturally associated to these curvature/operator pairs.
\end{abstract}

\section{Introduction}

This paper draws its inspiration from an observation about the scalar curvature function introduced by Perelman [2002], with the goal of illustrating the connection between conformally covariant operators and the $\mathscr{W}$-functional of Perelman.

Let $\left(M^{n}, g\right)$ be a Riemannian manifold endowed with a smooth measure $m$, which we write as

$$
d m=e^{-f} d \operatorname{Vol}(g) .
$$

The Bakry-Emery Ricci tensor of the Riemannian measure space $\left(M^{n}, g, m\right)$ is

$$
\operatorname{Ric}^{m}(g)=\operatorname{Ric}_{\infty}^{m}(g)=\operatorname{Ric}(g)+\nabla^{2} f .
$$

Although typically attributed to Bakry and Emery [1985], this tensor was studied much earlier by Lichnerowicz [1970]. In this setting, Perelman [2002] introduced a notion of scalar curvature given by

$$
R^{m}(g)=R_{\infty}^{m}(g)=R(g)+2 \Delta f-|\nabla f|^{2} .
$$

When the measure $m$ is the canonical Riemannian measure, then $f \equiv 0$ and the generalized curvatures agree with their classical counterparts.

From the perspective of conformal geometry, the scalar curvature is naturally considered in conjunction with the conformal Laplacian, the linear second-order

Research of the first and third authors was partially supported by NSF Grant DMS-0758601.

Research of the second author was partially supported by NSF Grant DMS-0800084.

MSC2000: primary 53A30; secondary 58J05.

Keywords: conformally invariant operators, metric measure spaces. 
operator which describes how the scalar curvature transforms under a conformal change of metric. In our setting, if $\hat{g}=v^{4 /(n-2)} g$, then

$$
R(\hat{g})=\frac{4(n-1)}{n-2} v^{(n+2) /(n-2)} L_{g} v,
$$

where

$$
L=-\Delta+\frac{n-2}{4(n-1)} R(g) .
$$

Moreover, the conformal Laplacian is conformally covariant: writing $\hat{g}=e^{2 w} g$,

$$
L_{\hat{g}} \phi=e^{-(n+2) / 2 w} L_{g}\left(e^{(n-2) / 2 w} \phi\right) .
$$

The question naturally arises, is there a linear, conformally covariant differential operator associated to Perelman's scalar curvature? What are the corresponding transformation formulas?

The answer to the first question is, somewhat surprisingly, "yes": The operator is given by

$$
\mathscr{L}_{2, \infty}^{m}=-\Delta+2\langle\nabla f, \cdot\rangle+\frac{n+2}{4} R_{\infty}^{m}(g)
$$

(see Section 4.1). Moreover, if $\hat{g}=v^{-4 /(n+2)} g$ is a conformal metric, then

$$
R_{\infty}^{m}(\hat{g})=\frac{4(n-1)}{3 n-2} v^{-(n-2) /(n+2)} \mathscr{L}_{2, \infty}^{m} v .
$$

Writing $\hat{g}=e^{2 w} g$, the operator in (1-2) satisfies the covariance property

$$
\left(\mathscr{L}_{2, \infty}^{m}\right)_{\hat{g}} \phi=e^{(n-2) / 2 w}\left(\mathscr{L}_{2, \infty}^{m}\right)_{g}\left(e^{-(n+2) / 2 w} \phi\right) .
$$

Note the interesting comparison with the bidegree of the conformal Laplacian in (1-1).

Our first goal in this paper is to put the preceding formulas for $R_{\infty}^{m}$ and $\mathscr{L}_{2, \infty}^{m}$ into a broader context. That is, by adapting the construction of Graham-Jenne-MasonSparling [1992] to Riemannian measure spaces we prove the existence of a 1parameter family of conformally covariant operators, of which $\mathscr{L}_{2, \infty}^{m}$ is a particular example (i.e., $\alpha=2$ ). As a byproduct of this construction we define a new family of scalar curvature functions $R^{(m, \alpha)}$ generalizing Perelman's scalar curvature. Thus, for each value of the parameter $\alpha$, we have a pair $\left(R^{(m, \alpha)}, \mathscr{L}_{\alpha}^{m}\right)$ consisting of a scalar curvature function and covariant operator. The relationship between curvature and operator are completely analogous to the case of the scalar curvature/conformal Laplacian detailed above. We remark that the conformally invariant curvatures of [Chang et al. 2006] figure in this construction in an important way.

The second goal of this paper is to study the variational problem naturally associated to this new family $\left(R^{(m, \alpha)}, \mathscr{L}_{\alpha}^{m}\right)$. As we shall see in Section 3, the EulerLagrange equation can be subcritical, critical (as it is for the usual scalar curvature), 
or even supercritical, depending on the value of $\alpha$. In Section 4 we prove existence of extremals for the Lagrangian in the subcritical case. For the remaining cases existence seems to be a difficult issue.

In Section 5 we study another special case $(\alpha=1)$, and formulate a weighted $L^{2}$-eigenvalue problem. We then give a characterization of the Yamabe invariant as the solution of a mini-max problem for this eigenvalue. This result is directly inspired by Perelman's work; indeed, the Lagrangian associated to the operator $\mathscr{L}_{1}^{m}$ is (up to a constant) Perelman's entropy functional. Why it is that a Lagrangian which comes from a construction in conformal geometry should coincide with Perelman's functional - which characterizes gradient Ricci solitons - is somewhat mysterious. In some sense, Section 5 brings us full circle: what started with an observation about Perelman's scalar curvature brings us via the Graham-JenneMason-Sparling construction back to Perelman's work.

Some material in this paper was announced in [Chang et al. 2006]. See also the note (added in proof) on page 55.

\section{Conformally covariant operators on $R M$-spaces}

In this section we adapt the construction of Fefferman and Graham [1985] and Graham, Jenne, Mason and Sparling [Graham et al. 1992] to construct families of conformally covariant differential operators associated to an $R M$-space. As we shall see here and in Section 3, the conformally invariant scalar and Ricci curvatures of [Chang et al. 2006] arise naturally in these constructions.

Let $\left(M^{n}, g\right)$ be a Riemannian manifold of dimension $n \geq 2$. A metrically defined differential operator $\mathscr{A}=\mathscr{A}_{g}$ is said to be conformally covariant of bidegree $(a, b)$ if it obeys the following transformation under a conformal change of metric $\hat{g}=e^{2 w} g$ :

$$
\mathscr{A}_{\hat{g}}(\psi)=e^{-b w} \mathscr{A}_{g}\left(e^{a w} \psi\right)
$$

for some constants $a, b$ and all $\psi \in C^{\infty}\left(M^{n}\right)$. For example, when $n=2, \mathscr{A}_{g}=\Delta_{g}$ is conformally covariant with $a=0$ and $b=2$. More generally, when $n \geq 3$ the conformal Laplacian

$$
\mathscr{A}_{g}=L_{g}=-\Delta_{g}+\frac{n-2}{4(n-1)} R(g)
$$

is conformally covariant with $a=(n-2) / 2$ and $b=(n+2) / 2$.

In [Graham et al. 1992], conformally covariant operators $P_{k}$ were constructed for all positive integers $k$ when $n$ is odd, and for $1 \leq k \leq n / 2$ when $n$ is even, with $a=(n-2 k) / 2$ and $b=(n+2 k) / 2$. The principal part of $P_{k}$ is given by $(-\Delta)^{k}$; when $k=1$ then $P_{1}$ is just the conformal Laplacian. These operators were derived from the ambient metric construction of Fefferman and Graham which is briefly described below. Aside from their intrinsic interest, they have also played a role in 
[Fefferman and Graham 2002; Fefferman and Hirachi 2003; Graham and Zworski 2003]. Given an $R M$-space $\left(M^{n}, g, m\right)$, we can modify the method of [Graham et al. 1992] to derive a family of operators $\mathscr{A}_{g}^{m}$ satisfying

$$
\text { if } \hat{g}=e^{2 w} g, \text { then } \quad \mathscr{A}_{\hat{g}}^{m}(\psi)=e^{-b w} \mathscr{A}_{g}^{m}\left(e^{a w} \psi\right),
$$

for some constants $a, b$, and for all $\psi \in C^{\infty}\left(M^{n}\right)$.

Theorem 2.1. Let $\left(M^{n}, g, m\right)$ be an $R M$-space with $n \geq 3$. Let $k$ be a positive integer; if $n$ is even we assume in addition that $1 \leq k \leq n / 2$. For $\alpha \in \mathbb{R}$, denote $\beta_{k}(\alpha)=(n \alpha-n+2 k) / 2$. Then, given any $\alpha \in \mathbb{R}$ there is an operator $P_{\alpha, k}^{m}$ satisfying (2-3) with $a=-\beta_{k}(\alpha)$ and $b=2 k-\beta_{k}(\alpha)$, the leading term of which is given by

$$
P_{\alpha, k}^{m}=\left(-\Delta_{g}+\alpha\langle\nabla f, \nabla \cdot\rangle\right)^{k}+\cdots
$$

When $\alpha=0$ the operator $P_{\alpha, k}^{m}$ coincides with $P_{k}$. For $k=1$ we have the formula

$$
P_{\alpha, k}^{m}(\psi)=-\Delta_{g} \psi+\alpha\langle\nabla f, \nabla \psi\rangle+\frac{n-2-n \alpha}{2(n-2)}\left(\alpha \Delta_{g} f+\frac{n \alpha+n-2}{2(n-1)} R(g)\right) \psi
$$

As in the work of Graham, Jenne, Mason and Sparling [Graham et al. 1992], our operators are constructed by an inductive algorithm; when $k$ becomes large the formulas become increasingly complicated. In fact, these authors presented two (equivalent) ways of deriving their operators. We will briefly describe one of their methods, indicating the modifications necessary to produce the measure-dependent operators $P_{\alpha, k}^{m}$.

To begin, given a Riemannian manifold $\left(M^{n}, g\right)$, let $\mathscr{G} \subset S^{2} T^{*} M^{n}$ denote the ray bundle consisting of metrics in the conformal class of $g$. Fixing a representative $g \in[g]$ determines a fiber variable $t$ on $\mathscr{G}$, by writing a general point in $\mathscr{G}$ in the form $\left(x, t^{2} g(x)\right)$. If $\left\{x^{i}\right\}$ are local coordinates on $M^{n}$, the coordinate system $\left(t, x^{i}\right)$ on $\varphi$ extends to a coordinate system $\left(t, x^{i}, \rho\right)$ on $\tilde{\mathscr{G}}=\mathscr{G} \times(-1,1)$, where $\rho$ is a defining function for $\mathscr{G}$, homogeneous of degree 0 (see [Fefferman and Graham 1985] for details). Using these coordinates we can define the ambient metric $\tilde{g}$ on $\tilde{\mathscr{G}}$ by

$$
\tilde{g}=2 \rho d t^{2}+2 t d t d \rho+t^{2} g_{i j}(x, \rho) d x^{i} d x^{j},
$$

where $g_{i j}(x, 0)=g_{i j}(x)$ is the given representative of $[g]$. For $\rho \neq 0$ the Taylor expansion of $g_{i j}(x, \rho)$ is determined by formally solving the Einstein equation

$$
\operatorname{Ric}(\tilde{g})=0 .
$$

We remark that in the construction of [Fefferman and Graham 1985], when $\mathrm{n}$ is even, (2-6) determines the Taylor coefficient of $g_{i j}$ up to the $(\rho)^{n / 2}$ term; the trace part of $g^{i j}\left(\partial_{\rho}\right)^{n / 2} g_{i j}$ is determined at $\rho=0$ but the trace-free part of $\left(\partial_{\rho}\right)^{n / 2} g_{i j}$ is not. When $n$ is odd, (2-6) determines the expansion of all orders. This partially 
explains the constraint on the order $k$ for the existence part of the GJMS operator when the dimension $n$ is even.

Let $\delta_{s}: \mathscr{G} \rightarrow \mathscr{G}$ denote the dilations $\delta_{s}(g)=s^{2} g$, with $s>0$. Functions on $\mathscr{G}$ which are homogeneous of degree $\beta$ with respect to $\delta_{s}$ are known as conformal densities of weight $\beta$. Given a density $\phi$ of weight $\beta$, consider the problem of extending $\phi$ to a harmonic function on $\tilde{\mathscr{G}}$ with the same homogeneity. That is, we want to find the formal power series solution of

$$
\tilde{\Delta}\left(t^{\beta} \phi\right)=0 .
$$

The operators of [Graham et al. 1992] arise as the obstruction to formally solving (2-7) with $\beta+(1 / 2) n=k=1,2,3, \ldots$

Given an $R M$-space $\left(M^{n}, g, m\right)$ we can also construct the ambient metric $\tilde{g}$, but we need to extend the density function $f$ associated to $m$ as well.

Lemma 2.2. Let $\left(M^{n}, g, m\right)$ be a Riemannian measure space with

$$
d m=e^{-f} d \operatorname{Vol}(g) .
$$

Let $k$ be a positive integer; if $n$ is even we assume in addition that $1 \leq k<n / 2$. Then there is an extension $\tilde{f}: \tilde{G} \rightarrow \mathbb{R}$ with

$$
\tilde{f}(t, x, \rho)=f(x, \rho)+n \log t,
$$

such that $f(x, 0)=f(x)$ for all $x \in M^{n}$, and $\tilde{f}$ satisfies

$$
\tilde{\Delta} \tilde{f}=O\left(\rho^{k}\right)
$$

near $\mathscr{G}$ on $\tilde{\mathscr{G}}$.

This lemma is a special case of Proposition 2.2 in [Graham et al. 1992]; see also Lemma 2.1 in [Fefferman and Hirachi 2003]. In order to make the paper selfcontained and to derive specific formulas for $P_{\alpha, k}^{m}$ in (2-4) for the case $k=1$, we will outline the proof here.

Proof. We will establish (2-9) by induction on $k$. Given a function $\psi$ defined on the ambient space $\psi=\psi(t, x, \rho)$, denote $\psi^{\prime}=\partial \psi / \partial \rho, \psi^{\prime \prime}=\partial^{2} \psi / \partial \rho^{2}$. Then

$$
\tilde{\Delta} \psi=t^{-2}\left(\Delta_{g} \psi+(n-2) \psi^{\prime}-2 \rho \psi^{\prime \prime}+2 t \partial_{t} \psi^{\prime}+\frac{1}{2} t g^{i j} g_{i j}^{\prime} \partial_{t} \psi-\rho(\log |g|)^{\prime} \psi^{\prime}\right),
$$

where $g=g_{i j}(x, \rho) d x_{i} d x_{j}$. Thus, for a function $\tilde{f}(t, x, \rho)=f(x, \rho)+n \log t$ with $f(x, 0)=f(x)$ we have

$(2-10) \quad t^{2} \tilde{\Delta} \tilde{f}=\Delta_{g} \tilde{f}+(n-2) f^{\prime}-2 \rho f^{\prime \prime}+2 t \partial_{t} f^{\prime}+\frac{n}{2} g^{i j} g_{i j}^{\prime}-\rho(\log |g|)^{\prime} f^{\prime}$. 
To see that $\tilde{f}$ can be chosen to satisfy (2-9) for $k=1$ and all $n>2$, we use the identities

$$
\begin{aligned}
g_{i j}^{\prime}(x, 0)=2 P_{i j} & =\frac{2}{n-2}\left(R_{i j}-\frac{1}{2(n-1)} R g_{i j}\right), \\
\left.(\log |g|)^{\prime}\right|_{\rho=0} & =\frac{1}{n-1} R,
\end{aligned}
$$

where $R_{i j}$ and $R$ are respectively the Ricci and scalar curvature of the metric $g$. Substituting these into the formula (2-10), we see that (2-9) for $k=1$ is equivalent to finding $f(x, \rho)$, with

$$
f^{\prime}(x, 0)=-\frac{1}{n-2} \Delta_{g} f(x)-\frac{n}{2(n-1)(n-2)} R,
$$

which can easily be done.

To see that (2-9) can be solved for all $k$ with $1 \leq k<\frac{n}{2}$ if $n$ is even and for all $k$ when $n$ is odd, we apply the same strategy that appears in the construction of the operators in [Graham et al. 1992]. That is, we inductively differentiate $\tilde{\Delta} \tilde{f}$ exactly $(k-1)$-times w.r.t. $\rho$, then evaluate at $\rho=0$. For example, when $k=2$, using the identities in (2-11) and doing some routine calculations we obtain

$$
\begin{array}{rl}
\left.t^{2}(\tilde{\Delta} \tilde{f})^{\prime}\right|_{\rho=0}=-2 P^{i j} \nabla_{i} \nabla_{j} & f-\frac{1}{2(n-1)} \nabla_{j} R \nabla_{j} f \\
& +\Delta_{g} f^{\prime}+(n-4) f^{\prime \prime}-n P^{i j} P_{i j}-\frac{1}{n-1} R f^{\prime}
\end{array}
$$

From (2-13), it is clear that to solve (2-9) for $k=2$ and $n \neq 4$ one only needs to choose $f(x, \rho)$ with $f^{\prime \prime}(x, 0)$ satisfying

$$
\begin{aligned}
(n-4) f^{\prime \prime}(x, 0)=2 P^{i j} \nabla_{i} & \nabla_{j} f(x)+\frac{1}{2(n-1)} \nabla R \nabla f(x) \\
& -\Delta_{g} f^{\prime}(x, 0)+n P^{i j} P_{i j}(x)+\frac{1}{n-1} R f^{\prime}(x, 0),
\end{aligned}
$$

with $f^{\prime}(x, 0)$ satisfying Equation (2-12). We refer to [Graham et al. 1992] for the proof of the general $k$.

Proof of Theorem 2.1. To derive the operators $P_{\alpha, k}^{m}$ we replace (2-7) with

$$
-\tilde{\Delta}\left(t^{\beta} \phi\right)+\alpha\langle\tilde{\nabla} \tilde{f}, \tilde{\nabla} \phi\rangle=0,
$$

where $\phi=\phi(x, \rho)$ is any extension of a given function $\phi$ defined on $M$ and where $\tilde{f}$ is an extension of $f$ chosen according to Lemma 2.2. The operators $P_{\alpha, k}^{m}$ arise as the obstruction to formally solving (2-15) up to order $\rho^{k}$ independent of the extension $\phi=\phi(x, \rho)$ of $\phi$. We then find that a suitable choice of $\beta$ is $\beta=$ $\beta_{k}(\alpha)=(n \alpha-n+2 k) / 2$ for each $k \geq 1$ when $n$ is odd, and for $1 \leq k<n / 2$ when $n$ 
is even. As the proof is by induction on $k$ and very similar to the proof in [Graham et al. 1992] we will only give an outline.

Given a smooth function $\tilde{\phi}=\phi(t, x, \rho)$ defined on the ambient space $\tilde{\mathscr{G}}$, we define the operator

$$
\tilde{L}_{\alpha, \tilde{g}}^{m}(\tilde{\phi})=-\tilde{\Delta}(\tilde{\phi})+\alpha\langle\tilde{\nabla} \tilde{f}, \tilde{\nabla} \tilde{\phi}\rangle .
$$

Let $\phi, f \in C^{\infty}(M)$ and suppose $\phi(x, \rho)$ and $f(x, \rho)$ are smooth extensions defined on $\varphi_{\text {; i.e., }} \phi(x, 0)=\phi(x)$ and $f(x, 0)=f(x)$. Given $\beta \in \mathbb{R}$, denote $\tilde{\phi}(t, x, \rho)=$ $t^{\beta} \phi(x, \rho)$ and $\tilde{f}(t, x, \rho)=f(x, \rho)+n \log t ;$ then

$$
\begin{aligned}
\tilde{L}_{\alpha \tilde{g}}^{m}(\tilde{\phi})=t^{\beta-2}\left(2 \rho \phi^{\prime \prime}-\right. & \left(2 \beta+(n-2)-\frac{1}{n-1} \rho R-n \alpha\right) \phi^{\prime}-\Delta_{g} \phi \\
& \left.\quad-\frac{1}{2(n-1)} \beta R \phi+\alpha \beta \phi f^{\prime}+\alpha g^{i j} \nabla_{i} \phi \nabla_{j} f-2 \rho \alpha \phi^{\prime} f^{\prime}\right) .
\end{aligned}
$$

Therefore,

$$
\begin{aligned}
\left.t^{2-\beta} \tilde{L}_{\alpha, \tilde{g}}^{m}(\tilde{\phi})\right|_{\rho=0}=(n \alpha-(n-2)+2 \beta) & \phi^{\prime}-\Delta_{g} \phi \\
& -\frac{1}{2(n-1)} \beta R \phi+\alpha \beta \phi f^{\prime}+\alpha\left\langle\nabla_{g} \phi, \nabla_{g} t\right\rangle .
\end{aligned}
$$

Consequently, if we choose $\beta=\beta_{1}(\alpha)$ so that $n \alpha-(n-2)-2 \beta=0$, and choose $\tilde{f}$ to satisfy (2-12) in Lemma 2.2, the operator $P_{\alpha, 1}^{m}$ given by

$$
P_{\alpha, 1}^{m}(\phi)=\left.t^{2-\beta} L_{\alpha, \tilde{g}}^{m}(\tilde{\phi})\right|_{\rho=0}
$$

is well defined and satisfies covariance property

$$
\left(P_{\alpha, 1}^{m}\right)_{\hat{g}}(\phi)=e^{(\beta-2) w}\left(P_{\alpha, 1}^{m}\right)_{g}\left(e^{-\beta w} \phi\right)
$$

for all functions $\phi \in C^{\infty}(M)$, where $\hat{g}=e^{2 w} g$. Note in the formula of $P_{\alpha, 1}^{m}$ we should replace $f$ by $\hat{f}=f+n w$. The explicit formula for $P_{\alpha, 1}^{m}$ for the choice of $f^{\prime}$ in (2-12) is given by (2-4).

As before, for general $k$ the idea of the proof is to differentiate the term $\tilde{\mathscr{L}}_{\alpha, \tilde{g}}^{m}(\tilde{\phi})$ exactly $(k-1)$-times w.r.t. $\rho$ and inductively define the operators $P_{\alpha, k}^{m}$ in a similar fashion. We refer to [Graham et al. 1992] for details.

Remarks. 1. The conformally invariant curvatures of [Chang et al. 2006] can also be defined in terms of the extension $\tilde{f}$. For example, $R_{n}^{m}(g)$ is given by

$$
R_{n}^{m}(g)=-\left.\frac{(n-1)(n-2)}{n^{2}}|\tilde{\nabla} \tilde{f}|^{2}\right|_{M^{n}} .
$$

2. When $n$ is even, the operators of [Graham et al. 1992] exist up to $k \leq n / 2$, but our construction above only gives the existence of operators for $k<n / 2$ due to the choice of the extension $\tilde{f}$ in Lemma 2.2. However, when $k=1$ the preceding 
remark indicates a way of modifying our construction, as follows. First, note that one can add a multiple of $R_{n}^{m}(g)$ to the operator $P_{\alpha, 1}^{m}$ and obtain an operator with the same conformal covariance property. For example, if one adds the term $C R_{n}^{m}(g)$, with

$$
C=C(\alpha, n)=\frac{n^{2}}{4(n-1)(n-2)} \alpha \beta_{1}(\alpha),
$$

then the operator defined by

$$
\begin{aligned}
\tilde{L}_{\alpha, 1}^{m}(\phi)= & P_{\alpha, 1}^{m}(\phi)+C(\alpha, n) R_{n}^{m}(g) \phi \\
=-\Delta_{g} \phi+\alpha\left\langle\nabla_{g} f,\right. & \left.\nabla_{g} \phi\right\rangle \\
& \quad-\beta_{1}\left(\left(1-\frac{n \alpha}{2}\right) \frac{1}{2(n-1)} R_{g}-\frac{\alpha}{2} \Delta_{g} f+\frac{\alpha}{4}\left|\nabla_{g} f\right|^{2}\right) \phi
\end{aligned}
$$

satisfies the conformal covariance property (2-3), with $a=-\beta_{1}(\alpha)$ and $b=2-$ $\beta_{1}(\alpha)$. It has the additional advantage that it exists for all $n \geq 2$, including $n=2$. When $k \geq 2$, it is not yet clear how to modify the operator $P_{\alpha, k}^{m}$. On the other hand, the existence of $m$-conformally covariant operators for all $k$ when $n$ is even and for $1 \leq k \leq \frac{n}{2}$ (when $n$ is odd) follows from an observation of R. Graham. The details are given in the next remark.

3. R. Graham pointed out to us another possible construction of conformally covariant operators on $R M$-spaces, by using the operators $P_{k}$ of [Graham et al. 1992]. Letting

$$
G_{\alpha, k}^{m}(\phi)=e^{\alpha f / 2} P_{k}\left(e^{-\alpha f / 2} \phi\right),
$$

it is easy to see that these operators satisfy the same conformal covariance as the operators $P_{\alpha, k}^{m}$ in Theorem 2.1. Interestingly, in general $G_{\alpha, k}^{m}$ and $P_{\alpha, k}^{m}$ do not agree. For example, when $k=1$ they again differ by a multiple $C_{\alpha, n}$ of $R_{n}^{m}(g)$.

\section{Properties of the operators}

In this section we will discuss some properties of the operators constructed in Section 2. To simplify the presentation, we will restrict ourselves to a discussion of the case $k=1$.

As before, $\left(M^{n}, g, d m\right)$ will be an $R M$-space, and $d m=e^{-f} d v_{g}$ defines the density function $f$. Let us define

$$
\begin{aligned}
\mathscr{L}_{\alpha}^{m} \psi & =P_{\alpha, k=1}^{m} \psi \\
& =-\Delta_{g} \psi+\alpha\langle\nabla f, \nabla \psi\rangle+\frac{n-2-n \alpha}{2(n-2)}\left(\alpha \Delta_{g} f+\frac{n \alpha+n-2}{2(n-1)} R(g)\right) \psi .
\end{aligned}
$$

We begin by summarizing some elementary properties of the operators $\mathscr{L}_{\alpha}^{m}$. 
Proposition 3.1. Let $\alpha \in \mathbb{R}$.

(i) $\mathscr{L}_{\alpha}^{m}$ is self-adjoint with respect to the measure

$$
d m_{\alpha}=e^{-\alpha f} d \operatorname{Vol}(g) .
$$

(ii) Suppose $\hat{g}=e^{2 w} g$ is a conformal metric. Then

$$
\left(\mathscr{L}_{\alpha}^{m}\right)_{\hat{g}}(\phi)=e^{(\beta(\alpha)-2) w} \mathscr{L}_{\alpha}^{m}\left(e^{-\beta(\alpha) w} \phi\right)
$$

for all $\phi \in C^{\infty}(M)$, where

$$
\beta(\alpha)=\frac{n \alpha-n+2}{2} .
$$

(iii) Denote $v=v_{\alpha}=e^{-\beta(\alpha) w}$. Then

$$
\left(\mathscr{L}_{\alpha}^{m}\right)_{\hat{g}}(1)=v^{-\gamma_{\alpha}}\left(\mathscr{L}_{\alpha}^{m}\right)_{g}(v)
$$

where

$$
\gamma_{\alpha}=\frac{n+2-n \alpha}{n-2-n \alpha}, \quad \alpha \neq \frac{n-2}{n} .
$$

Proof. Properties (i)-(iii) follow from the properties of the operators $P_{\alpha, k=1}^{m}$ described in Section 2.

Remarks. 1. The properties of $\mathscr{L}_{\alpha}^{m}$ listed in Proposition 3.1 are shared by any operator which differs from $\mathscr{L}_{\alpha}^{m}(g)$ by a constant multiple of $R_{n}^{m}(g)$. In particular, the operators $\varphi_{\alpha}^{m}$ satisfy the same properties.

2. One can interpret Equation (3-5) as defining a a scalar curvature associated to the triple $(g, m, \alpha)$. Let

$$
R^{(m, \alpha)}=R^{(m, \alpha)}(g)=\frac{n-2-n \alpha}{n-2}\left(R(g)+\frac{2 \alpha(n-1)}{n-2+n \alpha} \Delta_{g} f\right) .
$$

We will refer to $R^{(m, \alpha)}$ as the $(g, m, \alpha)$-scalar curvature, or just the $\alpha$-scalar curvature if the context is clear. Note we can also write

$$
R^{(m, \alpha)}(g)=\frac{4(n-1)}{n-2+n \alpha} \mathscr{L}_{\alpha}^{m}(1) .
$$

By (3-5) and (3-8), given a conformal metric

$$
\hat{g}=e^{2 w} g=v^{4 /(n-\alpha n-2)} g,
$$

the $\alpha$-scalar curvature of $\hat{g}$ is given by

$$
R^{(m, \alpha)}(\hat{g})=\frac{4(n-1)}{n-2+n \alpha} v^{-\gamma_{\alpha}}\left(\mathscr{L}_{\alpha}^{m}\right)_{g}(v) .
$$


These formulas define a pair $\left(R^{(m, \alpha)}, \mathscr{L}_{\alpha}^{m}\right)$ generalizing the well known example of the scalar curvature/conformal Laplacian $(R, L)$. Indeed, the pair $(R, L)$ is just $\left(R^{(m, 0)}, \mathscr{L}_{0}^{m}\right)$, i.e., the case $\alpha=0$.

3. It is interesting to note that the semilinear equation (3-10) associated to the $\alpha$-scalar curvature can be subcritical, critical, or super-critical with respect to the Sobolev embedding, depending on $\alpha$. To see this, we note the following apparent properties of the exponent $\gamma_{\alpha}$ :

(a) $\gamma_{0}=(n+2) /(n-2), \gamma_{1}=-1, \gamma_{(n+2) / n}=0$.

(b) $\lim _{\alpha \rightarrow \pm \infty} \gamma_{\alpha}=1$.

(c) $d \gamma_{\alpha} / d \alpha=4 n /(n-2-n \alpha)^{2}, \alpha \neq(n-2) / n$.

(d) $\lim _{\alpha \rightarrow((n-2) / n)^{-}} \gamma_{\alpha}=+\infty$.

(e) $\lim _{\alpha \rightarrow((n-2) / n)^{+}} \gamma_{\alpha}=-\infty$.

Here is a plot of $\gamma_{\alpha}$ as a function of $\alpha$ :

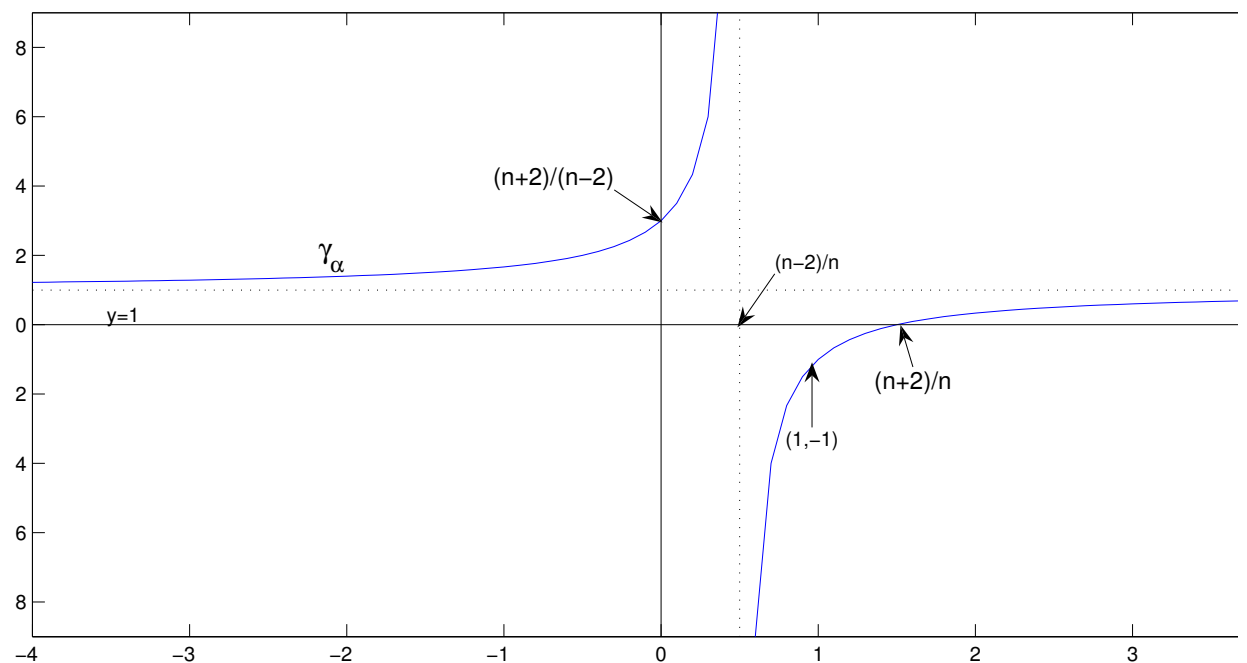

4. When $\alpha=(n-2) / n$ one needs to modify the definition of the $\alpha$-scalar curvature, since the definition (3-7) gives zero. In addition, one sees from the figure above that the exponent in (3-10) becomes infinite. Using an ansatz due to Branson known as continuation in the dimension, we can construct an operator $T^{m}$ to supplant

$$
\mathscr{L}_{(n-2) / n}^{m}=-\Delta+\frac{n-2}{n}\langle\cdot, \nabla f\rangle,
$$

and this permits us to define a scalar curvature $K^{m}(g)$ corresponding to the case $\alpha=(n-2) / n$. Indeed, set $\bar{\alpha}=(n-2) / n$, and define 


$$
\begin{aligned}
T_{g}^{m} \phi & =\lim _{\alpha \rightarrow \bar{\alpha}} \frac{1}{\beta(\alpha)}\left(\mathscr{L}_{\alpha}^{m}\left(e^{\beta(\alpha) \phi}\right)-\mathscr{L}_{\alpha}^{m}(1)\right) \\
= & \lim _{\alpha \rightarrow \bar{\alpha}} \frac{1}{\beta(\alpha)}\left(-\Delta\left(e^{\beta(\alpha) \phi}-1\right)+\alpha\left\langle\nabla\left(e^{\beta(\alpha) \phi}-1\right), \nabla f\right\rangle\right. \\
& \left.\quad-\frac{\beta}{(n-2)}\left(\alpha \Delta f+\frac{n \alpha+n-2}{2(n-1)} R\right)\left(e^{\beta(\alpha) \phi}-1\right)\right) \\
& =-\Delta w+\frac{n-2}{n}\langle\nabla w, \nabla f\rangle .
\end{aligned}
$$

We also define

$$
K^{m}(g)=\lim _{\alpha \rightarrow \bar{\alpha}} \frac{1}{\beta(\alpha)} \mathscr{L}_{\alpha}^{m}(1)=-\frac{1}{(n-1)}\left(R(g)+\frac{n-1}{n} \Delta f\right) .
$$

If $\hat{g}=e^{2 w} g$, then

$$
T_{\hat{g}}^{m}=e^{-2 w} T_{g}^{m},
$$

in analogy with the Laplacian on surfaces. Also, the behavior of $K^{m}$ under a conformal change is given by

$$
T_{g}^{m} w+c_{n} K^{m}(\hat{g}) e^{2 w}=c_{n} K^{m}(g),
$$

where $c_{n}=\frac{n-2}{n-1}$. Note the obvious parallel with the prescribed Gauss curvature equation.

As we observed in Remark 3 on the previous page, Equation (3-10) can be subcritical, critical, or supercritical depending on the value of $\alpha$. In the next Section we will study the existence of conformal metrics with constant $\alpha$-scalar curvature for the subcritical case; i.e., $-\infty<\alpha<0$ and $\alpha>1$.

\section{When $-\infty<\alpha<0$ or $\alpha>1$ : the subcritical cases}

In order to introduce the variational problems associated to the operators defined in Section 3 we define the functionals

$$
E_{\alpha}^{m}[v]=\int v \mathscr{L}_{\alpha}^{m} v d m_{\alpha}(g)=\left\langle v, \mathscr{L}_{\alpha}^{m} v\right\rangle_{L^{2}\left(d m_{\alpha}\right)},
$$

where

$$
d m_{\alpha}(g)=e^{-\alpha f} d \operatorname{Vol}(g) .
$$

We also define the constraint set

$$
\mathscr{C}_{\alpha}=\left\{v \in W^{1,2}(M) \mid v \geq 0, \int v^{\gamma_{\alpha}+1} d m_{\alpha}(g)=1\right\} .
$$

Note that

$$
1+\gamma_{\alpha}=\frac{2 n(\alpha-1)}{n(\alpha-1)+2}
$$


which is positive when $-\infty<\alpha<0$ or $\alpha>1$.

Consider the variational problem

$$
\inf _{v \in \mathscr{C}_{\alpha}} E_{\alpha}^{m}[v] .
$$

By the identity (3-10) this is equivalent to the following geometric variational problem: define

$$
\begin{gathered}
\mathscr{R}^{(m, \alpha)}: g \mapsto \int R^{(m, \alpha)}(g) d m_{\alpha}(g), \\
\mathscr{C}_{\alpha}([g])=\left\{\hat{g}=v^{4 /(n-\alpha n-2)} g \mid v \in C^{\infty}(M), v>0, v \in \mathscr{C}_{\alpha}\right\} .
\end{gathered}
$$

Then

$$
\mathscr{R}^{(m, \alpha)}[\hat{g}]=\frac{4(n-1)}{n-2+n \alpha} E_{\alpha}^{m}[v],
$$

where $\hat{g}=v^{4 /(n-\alpha n-2)} g$. Consequently,

$$
\begin{aligned}
& \inf _{\hat{g} \in \mathscr{C}_{\alpha}([g])} \mathscr{R}^{(m, \alpha)}[\hat{g}]=\frac{4(n-1)}{n-2+n \alpha} \inf _{v \in \mathscr{C}_{\alpha}} E_{\alpha}^{m}[v] \quad \text { if } \alpha<\frac{n-2}{n}, \\
& \sup _{\hat{g} \in \mathscr{C}_{\alpha}([g])} \mathscr{R}^{(m, \alpha)}[\hat{g}]=\frac{4(n-1)}{n-2+n \alpha} \inf _{v \in \mathscr{C}_{\alpha}} E_{\alpha}^{m}[v] \quad \text { if } \alpha>\frac{n-2}{n} .
\end{aligned}
$$

Again, when $\alpha=0$ we recover the familiar relation between the total scalar curvature and the Yamabe quotient. Moreover, when $\alpha<0$ or $\alpha>1$, the exponent in the definition of the constraint set $\mathscr{C}_{\alpha}$ is subcritical for the Sobolev embedding.

Theorem 4.1. (i) Suppose $\alpha \leq 0$ or $\alpha>1$. Then

$$
\inf _{v \in \mathscr{C}_{\alpha}} E_{\alpha}^{m}[v]>-\infty
$$

(ii) If $\alpha<0$ or $\alpha>1$, then the infimum in (4-10) is attained by a positive function $v=v_{\alpha} \in C^{\infty}(M)$ satisfying

$$
\mathscr{L}_{\alpha}^{m} v_{\alpha}=c v^{\gamma_{\alpha}}
$$

for some constant c. Equivalently:

- If $\alpha<0$ there is a conformal metric $\hat{g}=v_{\alpha}^{4 /(n-\alpha n-2)} g \in \mathscr{C}_{\alpha}([g])$ attaining the infimum of $\mathscr{R}^{m, \alpha}$.

- If $\alpha>1$ there is a conformal metric $\hat{g}=v_{\alpha}^{4 /(n-\alpha n-2)} g \in \mathscr{C}_{\alpha}([g])$ attaining the supremum of $\mathscr{R}^{m, \alpha}$.

In both cases, the $\alpha$-scalar curvature of $\hat{g}$ satisfies

$$
R^{(m, \alpha)}(\hat{g})=c .
$$


Proof. To verify (4-10), let $v \in \mathscr{C}_{\alpha}$; then

$$
E_{\alpha}^{m}[v] \geq \int|\nabla v|^{2} d \operatorname{Vol}(g)-C(g, f) \int v^{2} d \operatorname{Vol}(g) .
$$

Therefore, by the Sobolev embedding theorem,

(4-13) $\left(\int v^{2 n /(n-2)} d \operatorname{Vol}(g)\right)^{(n-2) / n} \leq C\|v\|_{W^{1,2}} \leq C\left(E_{\alpha}^{m}[v]+\int v^{2} d \operatorname{Vol}(g)\right)$.

When $\alpha \leq 0$, then $1+\gamma_{\alpha}$ satisfies

$$
2<1+\gamma_{\alpha} \leq \frac{2 n}{n-2}
$$

and by Hölder's inequality

$$
\int v^{2} d V \leq\left(\int v^{1+\gamma_{\alpha}} d V\right)^{2 /\left(1+\gamma_{\alpha}\right)} \leq C .
$$

It follows from (4-13) that

$$
E_{\alpha}^{m}[v] \geq C\|v\|_{2 n /(n-2)}^{2}-C \geq-C .
$$

If $\alpha>1$, by Hölder's inequality gives

$$
\int v^{2} d V \leq\left(\int v^{2 n /(n-2)} d V\right)^{\theta}\left(\int v^{1+\gamma_{\alpha}} d V\right)^{1-\theta},
$$

where

$$
\theta=\frac{n-2}{\alpha n}<\frac{n-2}{n} .
$$

Substituting this into (4-13) and using the constraint one verifies that (4-16) also holds for $\alpha>1$.

For existence, we now suppose $\alpha<0$ or $\alpha>1$, and let $\left\{v_{k}\right\}$ be a minimizing sequence for $E_{\alpha}^{m}$ with $v_{k} \in \mathscr{C}_{\alpha}$. We may assume

$$
E_{\alpha}^{m}\left[v_{k}\right] \leq \inf _{\mathscr{C}_{\alpha}} E_{\alpha}^{m}+1 .
$$

By (4-16) we see that

$$
\left\|v_{k}\right\|_{2 n /(n-2)} \leq C,
$$

and from (4-12) we conclude that $\left\{v_{k}\right\}$ is bounded in $W^{1,2}$. Since

$$
1+\gamma_{\alpha}<\frac{2 n}{n-2}
$$

when $\alpha<0$ or $\alpha>1$, the embedding

$$
W^{1,2} \hookrightarrow L^{1+\gamma_{\alpha}}
$$


is compact. Therefore, a subsequence of $\left\{v_{k}\right\}$ will converge weakly in $W^{1,2}$, but strongly in $L^{1+\gamma_{\alpha}}$, to a minimizer $v \in \mathscr{C}_{\alpha}$. Using the fact that $\mathscr{L}_{\alpha}^{m}$ is self-adjoint it is easy to check that a $W^{1,2}$-critical point of $E_{\alpha}^{m}$ subject to the constraint in (4-2) will satisfy (4-11) weakly. Elliptic regularity implies $v \in C^{\infty}$.

4.1. $\alpha=2$ : Perelman's scalar curvature. The case $\alpha=2$ is of particular interest. Note that

$$
R^{(m, 2)}(g)=-\frac{n+2}{n-2}\left(R(g)+\frac{4(n-1)}{3 n-2} \Delta f\right) .
$$

Recall the definition of the conformally invariant scalar curvature in [Chang et al. 2006]:

$$
R_{n}^{m}(g)=R(g)+\frac{2(n-1)}{n} \Delta f-\frac{(n-1)(n-2)}{n^{2}}|\nabla f|^{2} .
$$

When $n=\infty$, this corresponds formally to the scalar curvature introduced by Perelman [2002]:

$$
R_{\infty}^{m}(g)=R(g)+2 \Delta f-|\nabla f|^{2} .
$$

Comparing these formulas we see that

$$
R^{(m, 2)}(g)=\frac{(n+2)(n-1)}{3 n-2} R_{\infty}^{m}(g)-\frac{n^{2}(n+2)}{(3 n-2)(n-2)} R_{n}^{m}(g) .
$$

In particular, if we define the operator

$$
\mathscr{L}_{2, \infty}^{m}=\mathscr{L}_{2}^{m}+\frac{n^{2}(n+2)}{4(n-1)(n-2)} R_{n}^{m}(g),
$$

then by Remark 1 following Proposition 3.1, $\mathscr{L}_{2, \infty}^{m}$ enjoys the same conformal covariance properties as $\mathscr{L}_{2}^{m}$. One can check that

$$
\mathscr{L}_{2, \infty}^{m}=-\Delta+2\langle\nabla \cdot, \nabla f\rangle+\frac{n+2}{4} R_{\infty}^{m}(g),
$$

so that the "scalar curvature" associated to $\mathscr{L}_{2, \infty}^{m}$ is a multiple of Perelman's scalar curvature. This leads to the following corollary of Theorem 4.1:

Corollary 4.2. Given an $R M$-space $(M, g, m)$, there is a conformal metric $\hat{g}=$ $v^{-4 /(n+2)} g$ with

$$
R_{\infty}^{m}(\hat{g})=\text { const } .
$$

Moreover, $v$ can be realized as the infimum of the functional

$$
E_{2, \infty}^{m}[\phi]=\int\left\langle\phi, \mathscr{L}_{2, \infty}^{m} \phi\right\rangle d m_{2}(g)
$$

subject to the constraint $\int \phi^{2 n /(n+2)} d m_{2}=1$. 


\section{The case $\alpha=1$ : Perelman's entropy functional}

For the borderline case $\alpha=1$, the parameter $\gamma_{-1}=-1$, and the measure $m_{-1}=m$. Also, the 1-scalar curvature is given by

$$
R^{(m, 1)}(g)=2 \mathscr{L}_{1}^{m}(1)=-\frac{2}{n-2}(\Delta f+R(g)) .
$$

It follows that the functional $\mathscr{R}^{(m, 1)}$ defined in (4-5) is

$$
\begin{aligned}
\mathscr{R}^{(m, 1)}[g] & =\int R^{(m, 1)}(g) d m=-\frac{2}{n-2} \int(R(g)+\Delta f) d m \\
& =-\frac{2}{n-2} \int\left(R(g)+|\nabla f|^{2}\right) d m .
\end{aligned}
$$

Up to a constant, this is precisely the entropy functional defined by Perelman [2002, $\S 1]$. The difficulty in studying the corresponding variational problem (4-4) is that the constraint set $\mathscr{C}_{\alpha}$ is not well defined when $\alpha=1$, since then $\gamma_{1}=-1$ (or, to be more precise, it does not impose any constraint). In this section we study a related eigenvalue problem inspired by Perelman's work and point out an interesting connection to the Yamabe invariant.

To begin, let us introduce the modified constraint set

$$
\mathscr{D}^{m}(g)=\left\{v \in W^{1,2}(M) \mid v \geq 0, \int v^{2} e^{-(2 / n) f} d m=1\right\} .
$$

In a slight abuse of notation we will write $\hat{g}=v^{-2} g \in \mathscr{D}^{m}(g)$ whenever $v>0, v \in$ $C^{\infty}\left(M^{n}\right)$, and $v \in \mathscr{D}^{m}(g)$.

A key property used in our analysis is that the functional $E_{1}^{m}$ enjoys a certain conformal covariance when restricted to $\mathscr{D}^{m}$. To explain this, let us modify our notation slightly to emphasize the dependence of $E_{1}^{m}$ on the choice of metric, and write

$$
E_{1}^{m}(g)[v]=E_{1}^{m}[v]=\left\langle v,\left(\mathscr{L}_{1}^{m}\right)_{g} v\right\rangle_{L^{2}(d m)} .
$$

Lemma 5.1. For all smooth functions $\rho>0, v \in W^{1,2}\left(M^{n}\right)$, we have

$$
\begin{gathered}
E_{1}^{m}(g)[v]=E_{1}^{m}\left(\rho^{2} g\right)[\rho v], \\
v \in \mathscr{D}^{m}(g) \Longleftrightarrow \rho v \in \mathscr{D}^{m}\left(\rho^{2} g\right) .
\end{gathered}
$$

Proof. To prove (5-5), we use the covariance of $\mathscr{L}_{1}^{m}$ given in Proposition 3.1(ii): If $\hat{g}=e^{2 w} g$, then

$$
\left(\mathscr{L}_{1}^{m}\right)_{e^{2 w}{ }_{g}} \phi=e^{-w}\left(\mathscr{L}_{1}^{m}\right)_{g}\left(e^{-w} \phi\right) .
$$


Taking $e^{w}=\rho$, this implies

$$
\begin{aligned}
E_{1}^{m}\left(\rho^{2} g\right)[\rho v] & =\int\left\langle\rho v,\left(\mathscr{L}_{1}^{m}\right)_{\rho^{2} g}(\rho v)\right\rangle d m=\int\left\langle\rho v, \rho^{-1}\left(\mathscr{L}_{1}^{m}\right)_{g}\left(\rho^{-1} \rho v\right)\right\rangle d m \\
& =\int\left\langle v,\left(\mathscr{L}_{1}^{m}\right)_{g} v\right\rangle d m=E_{1}^{m}(g)[v] .
\end{aligned}
$$

To prove (5-6), suppose $\rho>0$ is smooth and write

$$
d m=e^{-f_{\rho}} d \operatorname{Vol}\left(\rho^{2} g\right),
$$

where $f_{\rho}=f+n \log \rho$. Therefore,

$$
\int(\rho v)^{2} e^{-(2 / n) f} d m=\int(\rho v)^{2} e^{-(2 / n)(f+n \log \rho)} d m=\int(\rho v)^{2} e^{-(2 / n) f_{\rho}} d m .
$$

If follows that $v \in \mathscr{D}^{m}(g)$ if and only if $(\rho v) \in \mathscr{D}^{m}\left(\rho^{2} g\right)$, as claimed.

For simplicity we now adopt Perelman's notation and write

$$
\begin{aligned}
\mathscr{F}^{m}[g] & =-\frac{(n-2)}{2} \mathscr{R}^{(m, 1)}[g] \\
& =\int(R(g)+\Delta f) d m=\int\left(R(g)+|\nabla f|^{2}\right) d m .
\end{aligned}
$$

It will be convenient if we normalize the measure $m$ to have total mass one; let $\mathscr{P}$ denote the set of all such smooth probability measures on $M^{n}$.

Theorem 5.2. Let $\left(M^{n}, g\right)$ be a Riemannian manifold.

(i) For each $m \in \mathscr{P}$,

$$
\lambda(m,[g])=\sup _{\hat{g} \in \mathscr{D}^{m}(g)} \mathscr{F}^{m}[\hat{g}]
$$

is attained by some metric $\sigma_{m} \in[g]$ satisfying

$$
R\left(\sigma_{m}\right)+\Delta_{\sigma_{m}} f_{m}=\lambda(m,[g]) e^{-\frac{2}{n} f_{m}},
$$

where $f_{m}$ is the density function of $m$ relative to $\sigma_{m}$.

(ii) Let $Y\left(M^{n},[g]\right)$ denote the Yamabe invariant of $[g]$. Then

$$
\lambda_{*}([g])=\inf _{m \in \mathscr{P}} \lambda(m,[g])=Y\left(M^{n},[g]\right),
$$

and the infimum is attained by all Yamabe measures, i.e., measures $m \in \mathscr{P}$ such that

$$
d m=e^{-f_{Y}} d \operatorname{Vol}(g),
$$

with $g_{Y}=e^{-(2 / n) f_{Y}} g$ a Yamabe metric. 
Proof. (i) First, by (4-7) we have

$$
\mathscr{F}^{m}(\hat{g})=-\frac{n-2}{2} \mathscr{R}^{(m, 1)}(\hat{g})=-(n-2) E_{1}^{m}(g)(v),
$$

where $\hat{g}=v^{-2} g$. Therefore, the variational problem in (5-8) is equivalent to a weighted $L^{2}$-eigenvalue problem for the operator $\mathscr{L}_{1}^{m}$. It follows that there is a function $v \in C^{\infty}\left(M^{n}\right) \cap \mathscr{D}^{m}(g), v>0$ satisfying the Euler-Lagrange equation

$$
\mathscr{L}_{1}^{m} v=\mu v e^{-(2 / n) f},
$$

where

$$
\mu=\inf _{v \in \mathscr{D}^{m}(g)} E_{1}^{m}(g)[v]=-\frac{1}{n-2} \sup _{\hat{g} \in \mathscr{D}^{m}(g)} \mathscr{F}^{m}[\hat{g}]=-\frac{1}{n-2} \lambda(m,[g]) .
$$

Using (3-10), Equation (5-12) implies the metric $\sigma_{m}=v^{-2} g$ satisfies

$$
R^{(m, 1)}\left(\sigma_{m}\right)=-\frac{2}{n-2} \lambda(m,[g]) v^{2} e^{-\frac{2}{n} f} .
$$

Since $d \operatorname{Vol}\left(\sigma_{m}\right)=v^{-n} d \operatorname{Vol}(g)$, it follows that $f_{m}=f-n \log v$, hence

$$
v^{2}=e^{-(2 / n) f_{m}} e^{(2 / n) f} .
$$

Substituting this into (5-13) and using the definition in (5-1) we find

$$
R\left(\sigma_{m}\right)+\Delta_{\sigma_{m}} f_{m}=-\frac{n-2}{2} R^{(m, 1)}\left(\sigma_{m}\right)=\lambda(m,[g]) e^{-(2 / n) f_{m}},
$$

as claimed.

(ii) We will prove the statement through a series of claims.

Claim 1. For each $m \in \mathscr{P}$,

$$
\lambda_{*}([g]) \leq Y\left(M^{n},[g]\right) .
$$

Proof. Let $g_{Y}=\rho_{0}^{2} g$ denote a Yamabe metric in $[g]$ and $m_{Y}=d \operatorname{Vol}\left(g_{Y}\right)$ denote the Yamabe measure associated to $g_{Y}$. We will assume that $g_{Y}$ has been normalized to have unit volume, so that $d m_{Y}$ is a probability measure and

$$
R\left(g_{Y}\right)=Y\left(M^{n},[g]\right) .
$$

By the definitions above,

$$
\lambda\left(m_{Y},[g]\right)=\sup _{v^{-2} g \in \mathscr{D}^{m_{Y}}(g)} \mathscr{F}^{m_{Y}}\left[v^{-2} g\right]=\sup _{v \in \mathscr{D}^{m_{Y}}(g)}-(n-2) E_{1}^{m_{Y}}(g)[v] .
$$

By Lemma 5.1,

$$
E_{1}^{m_{Y}}(g)[v]=E_{1}^{m_{Y}}\left(\rho_{0}^{2} g\right)\left[\rho_{0} v\right]=E_{1}^{m_{Y}}\left(g_{Y}\right)\left[\rho_{0} v\right]
$$


and

$$
v \in \mathscr{D}^{m_{Y}}(g) \Longleftrightarrow w=\rho_{0} v \in \mathscr{D}^{m_{Y}}\left(g_{Y}\right)
$$

Thus,

$$
\lambda\left(m_{Y},[g]\right)=\sup _{w \in \mathscr{D}^{m} Y\left(g_{Y}\right)}-(n-2) E_{1}^{m_{Y}}\left(g_{Y}\right)[w] .
$$

Now,

$$
-(n-2) E_{1}^{m_{Y}}\left(g_{Y}\right)[w]=\int\left(-(n-2)|\nabla w|^{2}+\left(R\left(g_{Y}\right)+\Delta f_{Y}\right) w^{2}\right) d m .
$$

Since $m_{Y}=d \operatorname{Vol}\left(g_{Y}\right)$, the density function $f_{Y} \equiv 0$. Therefore,

$$
\begin{aligned}
\lambda\left(m_{Y},[g]\right) & =\sup _{w \in \mathscr{D}^{m_{Y}}\left(g_{Y}\right)}-(n-2) E_{1}^{m_{Y}}\left(g_{Y}\right)[w] \\
& =\sup _{w \in \mathscr{D}^{m} Y\left(g_{Y}\right)} \int\left(-(n-2)|\nabla w|^{2}+R\left(g_{Y}\right) w^{2}\right) d m \\
& \leq \sup _{w \in \mathscr{D}^{m} Y\left(g_{Y}\right)} R\left(g_{Y}\right) \int w^{2} d m_{Y}=R\left(g_{Y}\right)=Y\left(M^{n},[g]\right) .
\end{aligned}
$$

Claim 2. As in [Chang et al. 2006], define the conformally invariant functional

$$
\mathscr{S}^{m}[g]=\int R_{n}^{m}(g) e^{(2 / n) f} d m .
$$

Then for each $m \in \mathscr{P}$,

$$
\lambda(m,[g]) \geq \mathscr{S}^{m}\left[\sigma_{m}\right] .
$$

Proof. Recall from above the definition of $R_{n}^{m}(g)$ :

$$
R_{n}^{m}(g)=R(g)+\frac{2(n-1)}{n} \Delta f-\frac{(n-1)(n-2)}{n^{2}}|\nabla f|^{2} .
$$

Taking $g=\sigma_{m}$, and using (5-9), we have

$$
R_{n}^{m}\left(\sigma_{m}\right)=\frac{(n-2)}{n} \Delta f_{m}-\frac{(n-1)(n-2)}{n^{2}}\left|\nabla f_{m}\right|^{2}+\lambda(m,[g]) e^{-2 / n} .
$$

Therefore,

$$
\begin{aligned}
\mathscr{S}^{m}\left[\sigma_{m}\right] & =\int\left(\frac{n-2}{n} \Delta f_{m}-\frac{(n-1)(n-2)}{n^{2}}\left|\nabla f_{m}\right|^{2}+\lambda(m,[g]) e^{-2 / n}\right) e^{(2 / n) f_{m}} d m \\
& =\lambda(m,[g])-\frac{n-2}{n^{2}} \int\left|\nabla f_{m}\right|^{2} e^{(2 / n) f_{m}} d m,
\end{aligned}
$$

which implies (5-17). 
Claim 3. We have

$$
\inf _{m \in \mathscr{P}} \mathscr{S}^{m}[g]=Y\left(M^{n},[g]\right) .
$$

The infimum is achieved by a measure $m_{Y}$ if and only if $m_{Y}$ is a Yamabe measure.

Proof. Let $m \in \mathscr{P}$ with density function $f$. By (5-16) and (5-18),

$$
\begin{aligned}
\mathscr{S}^{m}[g] & =\int\left(R(g)+\frac{2(n-1)}{n} \Delta f-\frac{(n-1)(n-2)}{n^{2}}|\nabla f|^{2}\right) e^{(2 / n) f} d m \\
& =\int\left(R(g)+\frac{(n-1)(n-2)}{n^{2}}|\nabla f|^{2}\right) e^{-(n-2) f / n} d \operatorname{Vol}(g) .
\end{aligned}
$$

Let $g_{m}=e^{-(2 / n) f} g$; then (5-20) implies that

$$
\varphi^{m}[g]=\int R\left(g_{m}\right) d \operatorname{Vol}\left(g_{m}\right) .
$$

Since $m$ is a probability measure, $g_{m}$ has unit volume, and it follows that

$$
\inf _{m \in \mathscr{P}} \mathscr{P}^{m}[g]=\inf _{g_{m}=e^{-(2 / n) f} g} \int R\left(g_{m}\right) d \operatorname{Vol}\left(g_{m}\right)=Y\left(M^{n},[g]\right) \text {. }
$$

Combining (5-17) and (5-19), we see that for any $m \in \mathscr{P}$,

$$
\lambda(m,[g]) \geq \mathscr{S}^{m}\left[\sigma_{m}\right] \geq \inf _{m \in \mathscr{P}} \mathscr{S}^{m}[g]=Y\left(M^{n},[g]\right) .
$$

Therefore,

$$
\lambda_{*}([g]) \geq Y\left(M^{n},[g]\right) .
$$

Combining this with (5-14), we arrive at (5-10).

Moreover, it is clear from the proofs of the claims that any Yamabe measure attains $\lambda_{*}([g])$.

Note added in proof. After this paper was accepted for publication, we learned about the preprints [Case 2010; 2011], which develop a variant of the conformal Laplacian for metrics with a measure.

\section{References}

[Bakry and Émery 1985] D. Bakry and M. Émery, "Diffusions hypercontractives”, pp. 177-206 in Séminaire de Probabilités XIX (Strasbourg, 1983/84), edited by J. Azéma and M. Yor, Lecture Notes in Math. 1123, Springer, Berlin, 1985. MR 88j:60131 Zbl 0561.60080

[Case 2010] J. S. Case, "The energy of a conformally warped manifold and applications", preprint, 2010. arXiv 1011.2728

[Case 2011] J. S. Case, "Conformally warped manifolds, quasi-Einstein metrics, and tractors", preprint, 2011. arXiv 1011.3009

[Chang et al. 2006] S.-Y. A. Chang, M. J. Gursky, and P. Yang, "Conformal invariants associated to a measure”, Proc. Natl. Acad. Sci. USA 103:8 (2006), 2535-2540. MR 2007e:53032 Zbl 1160.53356 
[Fefferman and Graham 1985] C. Fefferman and C. R. Graham, "Conformal invariants", pp. 95-116 in Élie Cartan et les mathématiques d'aujourd'hui: the mathematical heritage of Élie Cartan (Lyon, 1984), Astérisque, Société Mathématique de France, Paris, 1985. Special issue. MR 87g:53060 Zbl 0602.53007

[Fefferman and Graham 2002] C. Fefferman and C. R. Graham, " $Q$-curvature and Poincaré metrics”, Math. Res. Lett. 9:2 (2002), 139-151. MR 2003f:53053 Zbl 1016.53031

[Fefferman and Hirachi 2003] C. Fefferman and K. Hirachi, "Ambient metric construction of $Q$ curvature in conformal and CR geometries", Math. Res. Lett. 10:6 (2003), 819-831. MR 2005d: 53044 Zbl 1166.53309

[Graham and Zworski 2003] C. R. Graham and M. Zworski, "Scattering matrix in conformal geometry", Invent. Math. 152:1 (2003), 89-118. MR 2004c:58064 Zbl 1030.58022

[Graham et al. 1992] C. R. Graham, R. Jenne, L. J. Mason, and G. A. J. Sparling, "Conformally invariant powers of the Laplacian, I: Existence", J. London Math. Soc. (2) 46:3 (1992), 557-565. MR 94c:58226 Zbl 0726.53010

[Lichnerowicz 1970] A. Lichnerowicz, "Variétés riemanniennes à tenseur $C$ non négatif", $C . R$. Acad. Sci. Paris Sér. A-B 271 (1970), A650-A653. MR 42 \#3709 Zbl 0208.50003

[Perelman 2002] G. Perelman, "The entropy formula for the Ricci flow and its geometric applications", preprint, 2002. Zbl 1130.53001 arXiv math/0211159

Received June 18, 2009.

SUN-YUNG A. CHANG

chang@math.princeton.edu

DEPARTMENT OF MATHEMATICS

PRINCETON UNIVERSITY

Washington Road, Fine Hall

PRINCETON, NJ 08544

UNITED STATES

http://www.math.princeton.edu/ chang

MATTHEW J. GuRSKY

mgursky@nd.edu

DEPARTMENT OF MATHEMATICS

PRINCETON UNIVERSITY

WASHington RoAd, Fine Hall

PRINCETON, NJ 08544

UNITED STATES

PAUL YANG

yang@math.princeton.edu

DEPARTMENT OF MATHEMATICS

PRINCETON UNIVERSITY

Washington RoAd, Fine Hall

PRINCETON, NJ 08544

UNITED STATES 


\title{
PACIFIC JOURNAL OF MATHEMATICS
}

\author{
http://www.pjmath.org \\ Founded in 1951 by
}

E. F. Beckenbach (1906-1982) and F. Wolf (1904-1989)

\section{EDITORS}

V. S. Varadarajan (Managing Editor)

Department of Mathematics

University of California

Los Angeles, CA 90095-1555

pacific@math.ucla.edu

Vyjayanthi Chari

Department of Mathematics

University of California

Riverside, CA 92521-0135

chari@math.ucr.edu

\section{Robert Finn}

Department of Mathematics Stanford University

Stanford, CA 94305-2125

finn@math.stanford.edu

Kefeng Liu

Department of Mathematics

University of California

Los Angeles, CA 90095-1555

liu@math.ucla.edu
Darren Long

Department of Mathematics

University of California

Santa Barbara, CA 93106-3080

long@math.ucsb.edu

Jiang-Hua Lu

Department of Mathematics

The University of Hong Kong

Pokfulam Rd., Hong Kong jhlu@maths.hku.hk

Alexander Merkurjev

Department of Mathematics University of California

Los Angeles, CA 90095-1555 merkurev@math.ucla.edu
Sorin Popa

Department of Mathematics

University of California

Los Angeles, CA 90095-1555

popa@math.ucla.edu

Jie Qing

Department of Mathematics

University of California

Santa Cruz, CA 95064

qing@ cats.ucsc.edu

Jonathan Rogawski

Department of Mathematics

University of California

Los Angeles, CA 90095-1555

jonr@math.ucla.edu

\section{PRODUCTION}

pacific@math.berkeley.edu

Silvio Levy, Scientific Editor Matthew Cargo, Senior Production Editor

ACADEMIA SINICA, TAIPEI

CALIFORNIA INST. OF TECHNOLOGY

INST. DE MATEMÁTICA PURA E APLICADA

KEIO UNIVERSITY

MATH. SCIENCES RESEARCH INSTITUTE

NEW MEXICO STATE UNIV.

OREGON STATE UNIV.

\section{SUPPORTING INSTITUTIONS}

STANFORD UNIVERSITY
UNIV. OF BRITISH COLUMBIA
UNIV. OF CALIFORNIA, BERKELEY
UNIV. OF CALIFORNIA, DAVIS
UNIV. OF CALIFORNIA, LOS ANGELES
UNIV. OF CALIFORNIA, RIVERSIDE
UNIV. OF CALIFORNIA, SAN DIEGO
UNIV. OF CALIF., SANTA BARBARA

UNIV. OF CALIF., SANTA CRUZ

UNIV. OF MONTANA

UNIV. OF OREGON

UNIV. OF SOUTHERN CALIFORNIA

UNIV. OF UTAH

UNIV. OF WASHINGTON

WASHINGTON STATE UNIVERSITY

These supporting institutions contribute to the cost of publication of this Journal, but they are not owners or publishers and have no responsibility for its contents or policies.

See inside back cover or www.pjmath.org for submission instructions.

The subscription price for 2011 is US \$420/year for the electronic version, and \$485/year for print and electronic.

Subscriptions, requests for back issues from the last three years and changes of subscribers address should be sent to Pacific Journal of Mathematics, P.O. Box 4163, Berkeley, CA 94704-0163, U.S.A. Prior back issues are obtainable from Periodicals Service Company, 11 Main Street, Germantown, NY 12526-5635. The Pacific Journal of Mathematics is indexed by Mathematical Reviews, Zentralblatt MATH, PASCAL CNRS Index, Referativnyi Zhurnal, Current Mathematical Publications and the Science Citation Index.

The Pacific Journal of Mathematics (ISSN 0030-8730) at the University of California, c/o Department of Mathematics, 969 Evans Hall, Berkeley, CA 94720-3840, is published monthly except July and August. Periodical rate postage paid at Berkeley, CA 94704, and additional mailing offices. POSTMASTER: send address changes to Pacific Journal of Mathematics, P.O. Box 4163, Berkeley, CA 94704-0163.

PJM peer review and production are managed by EditFLOW ${ }^{\mathrm{TM}}$ from Mathematical Sciences Publishers.

PUBLISHED BY PACIFIC JOURNAL OF MATHEMATICS

at the University of California, Berkeley 94720-3840

A NON-PROFIT CORPORATION

Typeset in IATEX

Copyright $(2011$ by Pacific Journal of Mathematics 


\title{
PACIFIC JOURNAL OF MATHEMATICS
}

\author{
Volume $253 \quad$ No. $1 \quad$ September 2011
}

Singularities of the projective dual variety

ROLAND ABUAF

Eigenvalue estimates for hypersurfaces in $\mathbb{H}^{m} \times \mathbb{R}$ and applications

Pierre BÉRARD, Philippe CASTILlon and Marcos CaVAlCante

Conformal Invariants associated to a measure: Conformally covariant operators

Sun-Yung A. Chang, Matthew J. Gursky and Paul Yang

Compact symmetric spaces, triangular factorization, and Cayley coordinates

DEREK HABERMAS

Automorphisms of the three-torus preserving a genus-three Heegaard splitting

JESSE JOHNSON

The rationality problem for purely monomial group actions

HiDETAKA KiTAYAMA

On a Neumann problem with $p$-Laplacian and noncoercive resonant nonlinearity

Salvatore A. Marano and Nikolaos S. Papageorgiou

Minimal ramification in nilpotent extensions

NADYA MARKIN and STEPHEN V. ULLOM

Regularity of weakly harmonic maps from a Finsler surface into an $n$-sphere

XIAOHUAN MO and LIANG ZHAO

On the sum of powered distances to certain sets of points on the circle

NikOlai NikOlOV and RAFAEL RAFAilov

Formal geometric quantization II

PAUL-ÉMILE PARADAN

Embedded constant-curvature curves on convex surfaces

HAROLD ROSENBERG and MATTHIAS SCHNEIDER

A topological construction for all two-row Springer varieties 